\title{
Vitamin D Status in Postmenopausal Females in Saudi Arabia
}

\author{
$\underline{\text { Shatha Alharazy }}{ }^{1}$, Eman Alissa ${ }^{2}$, Adeel Chaudhary ${ }^{3}$, Susan Lanham-New ${ }^{4}$ and \\ M. Denise Robertson ${ }^{4}$ \\ ${ }^{1}$ Department of Physiology, Faculty of Medicine, King Abdulaziz University, Jeddah, Saudi Arabia., \\ ${ }^{2}$ Department of Clinical Biochemistry, Faculty of Medicine, King Abdulaziz University, Jeddah, Saudi Arabia., \\ ${ }^{3}$ Centre for Innovation and Personalized Medicine, Centre of Excellence in Genomic Medicine Research, King \\ Abdulaziz University, Jeddah, Saudi Arabia. and \\ ${ }^{4}$ Department of Nutritional Sciences, Faculty of Health and Medical Sciences, University of Surrey, Guilford, United \\ Kingdom
}

\begin{abstract}
Vitamin D (vitD) deficiency is highly prevalent in the Middle East (including Saudi Arabia) despite the abundance of sunlight. Older individuals in particular are at high risk of being vitD deficient. VitD binding protein (DBP), which acts as a carrier of vitD and its metabolites, has been reported to influence vitD status. In our study we aimed to investigate vitD status among postmenopausal women and its relation to DBP. A cross-sectional study was conducted at the King Fahad Medical Research Center, King Abdulaziz University, Jeddah, Saudi Arabia. Seventy six postmenopausal females (age $\geq 50$ years) who were not taking vitD supplementation and who were resident in Jeddah city, were randomly recruited from internal medicine clinics at King Abdulaziz University Hospital. Anthropometric measures, blood pressure, lifestyle history, dietary vitD intake and fasting blood samples were obtained from all study participants. Serum total 25 hydroxy-vitamin D $(25(\mathrm{OH}) \mathrm{D})$, DBP, albumin, parathyroid hormone, calcium, phosphate, magnesium and metabolic bone parameters were analysed. VitD deficiency was defined as serum total $25(\mathrm{OH}) \mathrm{D}$ level below $30 \mathrm{nmol} /$ L. The mean $( \pm \mathrm{SD})$ serum level of total $25(\mathrm{OH}) \mathrm{D}$ was $46.9 \pm 28.9 \mathrm{nmol} / \mathrm{L}$ with $36 \%$ of the study population being vitD deficient. Although non-significant, the vitD deficient group had lower DBP and higher dietary vitD intake levels when compared with those with serum vitD $>30 \mathrm{nmol} / \mathrm{L}$. In addition, DBP was inversely correlated with vitD dietary intake $(\mathrm{r}=-0.233$, $\mathrm{P}=0.046)$. In conclusion, vitD deficiency is highly prevalent among postmenopausal women living in Jeddah, Saudi Arabia. Intake of a vitD rich food seems to be associated with low DBP levels. Genetic polymorphisms in DBP will be studied in the future to find out a possible explanation for the differences in vitD status and DBP between individuals as well as the concomitant relationship between dietary vitD intake, DBP and serum 25(OH)D levels.
\end{abstract}

\section{Conflict of Interest}

There is no conflict of interest 